\title{
SOME RESULTS FOR $q$-POLY-BERNOULLI POLYNOMIALS WITH A PARAMETER
}

\author{
M. MECHACHA, M. A. BOUTICHE, M. RAHMANI, AND D. BEHLOUL
}

Received 27 October, 2017

\begin{abstract}
The main object of this paper is to investigate a new class of the generalized $q$-polyBernoulli numbers and polynomials with a parameter. We give explicit formulas and a recursive method for the calculation of the $q$-poly-Bernoulli numbers and polynomials. As a consequence, we derive a method for the calculation of the special values at negative integral points of the Arakawa-Kaneko zeta function also known as generalized Hurwitz zeta function.
\end{abstract}

2010 Mathematics Subject Classification: 11B68; 11B73

Keywords: Arakawa-Kaneko zeta function, Poly-Bernoulli numbers, recurrence relations, Stirling numbers

\section{INTRODUCTION}

Let $q$ be an indeterminate with $0 \leq q<1$. The $q$-analogue of $x$ is defined by

$$
[x]_{q}=\frac{1-q^{x}}{1-q}
$$

with $[0]_{q}=0$ and $\lim _{q \rightarrow 1}[x]_{q}=x$. Recently Komatsu in [12] introduced and studied a new family of polynomials, called $q$-poly-Bernoulli polynomials $B_{n, \rho, q}^{(k)}(z)$ with a real parameter $\rho$ which are defined by the following generating function:

$$
\begin{gathered}
F_{q, \rho}(t ; z):=\frac{\rho}{1-e^{-\rho t}} \operatorname{Li}_{k, q}\left(\frac{1-e^{-\rho t}}{\rho}\right) e^{-t z}=\sum_{n=0}^{\infty} B_{n, \rho, q}^{(k)}(z) \frac{t^{n}}{n !}, \\
(n \geq 0 ; k \in \mathbb{Z} ; \rho \neq 0)
\end{gathered}
$$

where $\operatorname{Li}_{k, q}(z)$ is the $q$-polylogarithm function [11] defined by

$$
\operatorname{Li}_{k, q}(z)=\sum_{n=1}^{\infty} \frac{z^{n}}{[n]_{q}^{k}} .
$$

Clearly, we have

$$
\lim _{q \rightarrow 1} B_{n, \rho, q}^{(k)}(z)=B_{n, \rho}^{(k)}(z)
$$


which is the poly-Bernoulli polynomial with a $\rho$ parameter [7], and

$$
\lim _{q \rightarrow 1} \operatorname{Li}_{k, q}(z)=\operatorname{Li}_{k}(z),
$$

which is the ordinary polylogarithm function, defined by

$$
\operatorname{Li}_{k}(z)=\sum_{m=1}^{\infty} \frac{z^{m}}{m^{k}}
$$

In addition, when $z=0, B_{n, \rho}^{(k)}(0)=B_{n, \rho}^{(k)}$ is the poly-Bernoulli number with a $\rho$ parameter. When $z=0$ and $\rho=1, B_{n, 1}^{(k)}(0)=B_{n}^{(k)}$ is the poly-Bernoulli number $[1-3,10]$ defined by

$$
\frac{\operatorname{Li}_{k}\left(1-e^{-t}\right)}{1-e^{-t}}=\sum_{n=0}^{\infty} B_{n}^{(k)} \frac{t^{n}}{n !},
$$

In this paper, we propose to investigate a new class of the generalized $q$-polyBernoulli numbers and polynomials with a parameter which we call $(m, q)$-polyBernoulli polynomials with a parameter $\rho$. We establish several properties of these polynomials. The study of $(m, q)$-poly-Bernoulli polynomials with a parameter yields an interesting algorithm for calculating $\mathbb{B}_{n, m}^{(k)}(z ; \rho, q)$. As an application, we derive a recursive method for the calculation of the special values at negative integral points of the Arakawa-Kaneko zeta function.

We first recall some basic definitions and some results $[8,16]$ that will be useful in the rest of the paper. The (signed) Stirling numbers $s(n, i)$ of the first kind are the coefficients in the following expansion:

$$
x(x-1) \cdots(x-n+1)=\sum_{i=0}^{n} s(n, i) x^{i}, \quad n \geq 1
$$

and satisfy the recurrence relation given by

$$
s(n+1, i)=s(n, i-1)-n s(n, i) \quad(1 \leq i \leq n) .
$$

The Stirling numbers of the second kind, denoted $S(n, i)$ are the coefficients in the expansion

$$
x^{n}=\sum_{i=0}^{n} S(n, i) x(x-1) \cdots(x-i+1), \quad n \geq 1 .
$$

These numbers count the number of ways to partition a set of $n$ elements into exactly $i$ nonempty subsets.

The exponential generating functions for $s(n, i)$ and $S(n, i)$ are given by

$$
\sum_{n=i}^{\infty} s(n, i) \frac{z^{n}}{n !}=\frac{1}{i !}[\ln (1+z)]^{i}
$$


and

$$
\sum_{n=i}^{\infty} S(n, i) \frac{z^{n}}{n !}=\frac{1}{i !}\left(e^{z}-1\right)^{i}
$$

respectively.

The weighted Stirling numbers $\delta_{n}^{i}(x)$ of the second kind are defined by (see [5,6])

$$
\begin{aligned}
\delta_{n}^{i}(x) & =\frac{1}{i !} \Delta^{i} x^{n} \\
& =\frac{1}{i !} \sum_{j=0}^{i}(-1)^{i-j}\left(\begin{array}{l}
i \\
j
\end{array}\right)(x+j)^{n},
\end{aligned}
$$

where $\Delta$ denotes the forward difference operator. The exponential generating function of $g_{n}^{k}(x)$ is given by

$$
\sum_{n=i}^{\infty} \delta_{n}^{i}(x) \frac{z^{n}}{n !}=\frac{1}{i !} e^{x z}\left(e^{z}-1\right)^{i}
$$

and weighted Stirling numbers $\delta_{n}^{i}(x)$ satisfy the following recurrence relation:

$$
8_{n+1}^{i}(x)=8_{n}^{i-1}(x)+(x+i) \delta_{n}^{i}(x) \quad(1 \leq i \leq n) .
$$

In particular, we have for nonnegative integer $r$

$$
f_{n}^{i}(0)=S(n, i) \text { and } f_{n}^{i}(r)=\left\{\begin{array}{l}
n+r \\
i+r
\end{array}\right\}_{r} .
$$

where $\left\{\begin{array}{c}n \\ i\end{array}\right\}_{r}$ denotes the $r$-Stirling numbers of the second kind [4].

\section{THE $(m, q)$-POLY-BERNOULLI NUMBERS WITH A PARAMETER $\rho$}

In order to compute $B_{n, \rho, q}^{(k)}:=B_{n, \rho, q}^{(k)}(0)$, we define $(m, q)$-poly-Bernoulli numbers $\mathbb{B}_{n, m}^{(k)}(\rho, q)$ with a parameter $\rho$ in terms of $m$-Stirling numbers of the second kind by:

$$
\mathbb{B}_{n, m}^{(k)}(\rho, q)=\frac{(-\rho)^{n}[m+1]_{q}^{k}}{m !} \sum_{i=0}^{n} \frac{(m+i) ! 8_{n}^{i}(m)}{(-\rho)^{i}[m+i+1]_{q}^{k}}, m \geq 0
$$

with $\mathbb{B}_{0, m}^{(k)}(\rho, q)=1$ and $\mathbb{B}_{n, 0}^{(k)}(\rho, q)=B_{n, \rho, q}^{(k)}$.

By direct computation from (2.1), we find

$$
\begin{aligned}
& \mathbb{B}_{0, m}^{(k)}(\rho, q)=1, \\
& \mathbb{B}_{1, m}^{(k)}(\rho, q)=(m+1)\left(\frac{q^{m+1}-1}{q^{m+2}-1}\right)^{k}-m,
\end{aligned}
$$




$$
\begin{aligned}
\mathbb{B}_{2, m}^{(k)}(\rho, q) & =(m+1)(m+2)\left(\frac{q^{m+1}-1}{q^{m+3}-1}\right)^{k} \\
& -\left(2 m^{2}+3 m+1\right)\left(\frac{q^{m+1}-1}{q^{m+2}-1}\right)^{k}+m^{2} .
\end{aligned}
$$

The following Theorem gives us a relation between the $(m, q)$-poly-Bernoulli numbers $\mathbb{B}_{n, m}^{(k)}(\rho, q)$ and $q$-poly-Bernoulli numbers $B_{n}^{(k)}(\rho, q):=B_{n, \rho, q}^{(k)}$.

Theorem 1. For $m \geq 0$, we have

$$
\mathbb{B}_{n, m}^{(k)}(\rho, q)=\frac{(-\rho)^{m}[m+1]_{q}^{k}}{m !} \sum_{i=0}^{m} s(m, i) \frac{B_{n+i}^{(k)}(\rho, q)}{(-\rho)^{i}} .
$$

Proof. The explicit formula (2.2) can be derived from a known result in [14, p. 681, Corollary 1] for the Stirling transform upon specializing the initial sequence

$$
a_{0, m}=\frac{m !}{(-\rho)^{m}[m+1]_{q}^{k}}
$$

The next Theorem contains the exponential generating function for $(m, q)$-polyBernoulli numbers with a parameter $\rho$.

Theorem 2. The exponential generating function for $\mathbb{B}_{n, m}^{(k)}(\rho, q)$ is given by

$$
\sum_{n=0}^{\infty} \mathbb{B}_{n, m}^{(k)}(\rho, q) \frac{t^{n}}{n !}=\frac{(-\rho)^{m+n}[m+1]_{q}^{k}}{m !} e^{m t}\left(e^{-t} \frac{d}{d t}\right)^{m} F_{q, \rho}(t ; z) .
$$

Proof. We have

$$
\begin{aligned}
\sum_{n=0}^{\infty} \mathbb{B}_{n, m}^{(k)}(\rho, q) \frac{t^{n}}{n !} & =\frac{(-\rho)^{m}[m+1]_{q}^{k}}{m !} \sum_{i=0}^{m} s(m, i) \sum_{n=0}^{\infty} \frac{B_{n+i}^{(k)}(\rho, q)}{(-\rho)^{i}} \frac{t^{n}}{n !} \\
& =\frac{(-\rho)^{m+n}[m+1]_{q}^{k}}{m !} \sum_{i=0}^{m} s(m, i) \frac{d^{i}}{d t^{i}}\left(\frac{\rho}{1-e^{t}} \operatorname{Li}_{k, q}\left(\frac{1-e^{t}}{\rho}\right)\right)
\end{aligned}
$$

Since [13]

$$
\sum_{i=0}^{m} s(m, i)\left(\frac{d}{d t}\right)^{i}=e^{m t}\left(e^{-t} \frac{d}{d t}\right)^{m},
$$

we get the desired result.

Next, we propose an algorithm, which is based on a three-term recurrence relation, for calculating the $(m, q)$-poly-Bernoulli numbers $\mathbb{B}_{n, m}^{(k)}(\rho, q)$ with a parameter $\rho$. 
Theorem 3. For every integer $k$, the $\mathbb{B}_{n, m}^{(k)}(\rho, q)$ satisfies the following three-term recurrence relation:

$$
\mathbb{B}_{n+1, m}^{(k)}(\rho, q)=(m+1)\left(\frac{q^{m+1}-1}{q^{m+2}-1}\right)^{k} \mathbb{B}_{n, m+1}^{(k)}(\rho, q)-\rho m \mathbb{B}_{n, m}^{(k)}(\rho, q)
$$

with the initial sequence given by $\mathbb{B}_{0, m}^{(k)}(\rho, q)=1$.

Proof. From (2.2) and (1.4), we have

$$
\mathbb{B}_{n, m+1}^{(k)}(\rho, q)=\frac{(-\rho)^{m+1}[m+2]_{q}^{k}}{(m+1) !} \sum_{i=0}^{m+1}(s(m, i-1)-m s(m, i)) \frac{B_{n+i}^{(k)}(\rho, q)}{(-\rho)^{i}} .
$$

After some simplifications, we find that

$$
\mathbb{B}_{n, m+1}^{(k)}(\rho, q)=\frac{1}{[m+1]_{q}^{k}} \frac{(-\rho)[m+2]_{q}^{k}}{m+1}\left(\frac{1}{(-\rho)} \mathbb{B}_{n+1, m}^{(k)}(\rho, q)-m \mathbb{B}_{n, m}^{(k)}(\rho, q)\right)
$$

This evidently equivalent to (2.3).

Remark 1. If we set $\rho=1, k=1$ and $q \rightarrow 1$, in (2.3), we get

$$
B_{n+1, m}=\frac{(m+1)^{2}}{(m+2)} B_{n, m+1}-m B_{n, m}
$$

an algorithm for the classical Bernoulli numbers with $B_{1}=\frac{1}{2}$. See [15] for the case $B_{1}=-\frac{1}{2}$.

\section{THE $(m, q)$-POLY-BERNOULLI POLYNOMIALS WITH A PARAMETER $\rho$}

For $m \geq 0$, let us consider the $(m, q)$-poly-Bernoulli polynomials with a parameter $\mathbb{B}_{n, m}^{(k)}(z ; \rho, q)$ as follows:

$$
\mathbb{B}_{n, m}^{(k)}(z ; \rho, q)=\sum_{i=0}^{n}(-1)^{n-i}\left(\begin{array}{l}
n \\
i
\end{array}\right) \mathbb{B}_{i, m}^{(k)}(\rho, q) z^{n-i} .
$$

It is easy to show that the generating function of $\mathbb{B}_{n, m}^{(k)}(z ; \rho, q)$ is given by

$$
\begin{aligned}
\sum_{n \geq 0} \mathbb{B}_{n, m}^{(k)}(z ; \rho, q) \frac{t^{n}}{n !}= & e^{-z t} \sum_{n \geq 0} \mathbb{B}_{n, m}^{(k)}(\rho, q) \frac{t^{n}}{n !} \\
& =\frac{1}{m !}(-\rho)^{m+n}[m+1]_{q}^{k} e^{(m-z) t}\left(e^{-t} \frac{d}{d t}\right)^{m} F_{q, \rho}(t ; z) .
\end{aligned}
$$

Next, we show an explicit formula about $\mathbb{B}_{n, m}^{(k)}(z ; \rho, q)$. 
Theorem 4. The following formula holds true

$$
\mathbb{B}_{n, m}^{(k)}(z ; \rho, q)=\frac{[m+1]_{q}^{k}}{m !} \sum_{i=0}^{n}(-\rho)^{n-i} \frac{(m+i) !}{[m+i+1]_{q}^{k}} \wp_{n}^{i}\left(\frac{z}{\rho}+m\right) .
$$

Proof. From (3.1), we have

$$
\begin{aligned}
\mathbb{B}_{n, m}^{(k)}(z ; \rho, q) & =\frac{[m+1]_{q}^{k}}{m !} \sum_{i=0}^{n}(-1)^{n-i}\left(\begin{array}{c}
n \\
i
\end{array}\right) \sum_{j=0}^{i} \frac{(-\rho)^{i-j}(m+j) ! 8_{i}^{j}(m)}{[m+j+1]_{q}^{k}} z^{n-i} \\
& =\frac{[m+1]_{q}^{k}}{m !} \sum_{j=0}^{n} \frac{(-\rho)^{n-j}(m+j) !}{[m+j+1]_{q}^{k}} \sum_{i=0}^{n}\left(\begin{array}{l}
n \\
i
\end{array}\right) \delta_{i}^{j}(m)\left(\frac{z}{\rho}\right)^{n-i} .
\end{aligned}
$$

By using the relation

$$
\wp_{n}^{i}(x+y)=\sum_{l=0}^{n}\left(\begin{array}{l}
n \\
l
\end{array}\right) \wp_{l}^{i}(x) y^{n-l}
$$

we obtain

$$
\mathbb{B}_{n, m}^{(k)}(z ; \rho, q)=\frac{[m+1]_{q}^{k}(-\rho)^{n}}{m !} \sum_{j=0}^{n} \frac{(m+j) !}{(-\rho)^{j}[m+j+1]_{q}^{k}} \S_{n}^{j}\left(\frac{z}{\rho}+m\right),
$$

we arrive at the desired result.

Theorem 5. The polynomials $\mathbb{B}_{n, m}^{(k)}(z ; \rho, q)$ satisfy the following three-term recurrence relation:

$$
\begin{aligned}
\mathbb{B}_{n+1, m}^{(k)}(z ; \rho, q)=(m+1)\left(\frac{q^{m+1}-1}{q^{m+2}-1}\right)^{k} \mathbb{B}_{n, m+1}^{(k)}(z ; \rho, q) & \\
& +(z-\rho m) \mathbb{B}_{n, m}^{(k)}(z ; \rho, q),
\end{aligned}
$$

with the initial sequence given by

$$
\mathbb{B}_{0, m}^{(k)}(z ; \rho, q)=1 .
$$

Proof. From (3.1), we get

$$
\begin{aligned}
\frac{d}{d z} \mathbb{B}_{n, m}^{(k)}(z ; \rho, q) & =\sum_{i=0}^{n}(n-i)(-1)^{n-i}\left(\begin{array}{l}
n \\
i
\end{array}\right) \mathbb{B}_{i, m}^{(k)}(\rho, q) z^{n-i-1} \\
& =n \sum_{i=0}^{n}(-1)^{n-i}\left(\begin{array}{c}
n \\
i
\end{array}\right) \mathbb{B}_{i, m}^{(k)}(\rho, q) z^{n-i-1} \\
& -n \sum_{i=1}^{n}(-1)^{n-i}\left(\begin{array}{c}
n-1 \\
i-1
\end{array}\right) \mathbb{B}_{i, m}^{(k)}(\rho, q) z^{n-i-1}
\end{aligned}
$$


Then

$$
\begin{aligned}
& z \frac{d}{d z} \mathbb{B}_{n, m}^{(k)}(z ; \rho, q)=n \mathbb{B}_{n, m}^{(k)}(z ; \rho, q) \\
& \quad-n \sum_{i=0}^{n-1}(-1)^{n-i-1}\left(\begin{array}{c}
n-1 \\
i
\end{array}\right) \mathbb{B}_{i+1, m}^{(k)}(\rho, q) z^{n-i-1} .
\end{aligned}
$$

Now, using (2.3), we have

$$
\begin{aligned}
z \frac{d}{d z} \mathbb{B}_{n, m}^{(k)}(z ; \rho, q) & =n \mathbb{B}_{n, m}^{(k)}(z ; \rho, q)+n \rho m \sum_{i=0}^{n-1}\left(\begin{array}{c}
n-1 \\
i
\end{array}\right) \mathbb{B}_{i, m}^{(k)}(\rho, q)(-z)^{n-i-1} \\
& -n(m+1)\left(\frac{q^{m+1}-1}{q^{m+2}-1}\right) \sum_{i=0}^{k-1}\left(\begin{array}{c}
n-1 \\
i
\end{array}\right) \mathbb{B}_{i, m+1}^{(k)}(\rho, q)(-z)^{n-i-1},
\end{aligned}
$$

which, after simplification, yields

$$
\begin{aligned}
z \mathbb{B}_{n-1, m}^{(k)}(z ; \rho, q)= & \mathbb{B}_{n, m}^{(k)}(z ; \rho, q)-(m+1)\left(\frac{q^{m+1}-1}{q^{m+2}-1}\right)^{k} \mathbb{B}_{n-1, m+1}^{(k)}(z ; \rho, q) \\
& +\rho m \mathbb{B}_{n-1, m}^{(k)}(z ; \rho, q),
\end{aligned}
$$

which is obviously equivalent to (3.2) and the proof is complete.

As consequence of Theorem 5, one can deduce a three-term recurrence relation for $(m, q)$-poly-Bernoulli polynomials with a parameter $\rho$ and negative upper indices $\mathbb{B}_{n, m}^{(-k)}(z ; \rho, q)$.

Corollary 1. The $\mathbb{B}_{n, m}^{(-k)}(z ; \rho, q)$ satisfies the following three-term recurrence relation:

$$
\begin{aligned}
\mathbb{B}_{n+1, m}^{(-k)}(z ; \rho, q)= & (m+1)\left(\frac{q^{m+2}-1}{q^{m+1}-1}\right)^{k} \mathbb{B}_{n, m+1}^{(-k)}(z ; \rho, q) \\
& +(z-\rho m) \mathbb{B}_{n, m}^{(-k)}(z ; \rho, q),
\end{aligned}
$$

with the initial sequence given by

$$
\mathbb{B}_{0, m}^{(-k)}(z ; \rho, q)=1
$$

The next result gives a method for the calculation of the special values at negative integral points of the Arakawa-Kaneko zeta function. Recall that the ArakawaKaneko zeta function $\xi_{k}(s, x)$, for $s \in \mathbb{C}, x>0, k \in \mathbb{Z}$, is defined by [9]

$$
\xi_{k}(s, x)=\frac{1}{\Gamma(s)} \int_{0}^{+\infty} t^{s-1} \frac{\operatorname{Li}_{k}\left(1-e^{-t}\right)}{1-e^{-t}} e^{-x t} d t
$$


It is well-known that the special values at negative integral points are given in terms of poly-Bernoulli polynomials

$$
\xi_{k}(-n, x)=(-1)^{n} B_{n}^{(k)}(x), n \geq 0 .
$$

We now present the following algorithm for $\xi_{k}(-n, x)$. We start with the sequence $\mathcal{K}_{0, m}=1$ as the first row of the matrix $\left(\mathcal{K}_{n, m}\right)_{n, m \geq 0}$. Each entry is determined recursively by

$$
\mathcal{K}_{n+1, m}(k, x)=\frac{(m+1)^{k+1}}{(m+2)^{k}} \mathcal{K}_{n, m+1}(k, x)+(x-m) \mathcal{K}_{n, m}(k, x) .
$$

Then

$$
\xi_{k}(-n, x)=(-1)^{n} \mathcal{K}_{n, 0}(k, x)
$$

where $\mathcal{K}_{n, 0}(k, x)$ are the first column of the matrix $\left(\mathcal{K}_{n, m}\right)_{n, m \geq 0}$.

\section{CONCLusion}

In our present research, we have investigated a new class of the generalized $q$ poly-Bernoulli numbers and polynomials with a parameter. As a consequence, we derive a method for the calculation of the special values at negative integral points of the Arakawa-Kaneko zeta function. We have also given a recursive method for the calculation of $q$-poly-Bernoulli numbers and polynomials with parameter.

\section{ACKNOWLEDGEMENTS}

The authors would like to thank an anonymous referee for the helpful comments.

\section{REFERENCES}

[1] T. Arakawa, T. Ibukiyama, and M. Kaneko, Bernoulli numbers and zeta functions. With an appendix by Don Zagier. Tokyo: Springer, 2014. doi: 10.1007/978-4-431-54919-2.

[2] A. Bayad and Y. Hamahata, "Polylogarithms and poly-Bernoulli polynomials." Kyushu J. Math., vol. 65, no. 1, pp. 15-24, 2011, doi: 10.2206/kyushujm.65.15.

[3] B. Bényi and P. Hajnal, "Combinatorics of poly-Bernoulli numbers." Stud. Sci. Math. Hung., vol. 52, no. 4, pp. 537-558, 2015, doi: 10.1556/012.2015.52.4.1325.

[4] A. Z. Broder, "The $r$-Stirling numbers." Discrete Math., vol. 49, pp. 241-259, 1984, doi: 10.1016/0012-365X(84)90161-4.

[5] L. Carlitz, "Weighted Stirling numbers of the first and second kind. I." Fibonacci Q., vol. 18, pp. $147-162,1980$.

[6] L. Carlitz, "Weighted Stirling numbers of the first and second kind. II." Fibonacci Q., vol. 18, pp. 242-257, 1980.

[7] M. Cenkci and T. Komatsu, "Poly-Bernoulli numbers and polynomials with a $q$ parameter." $J$. Number Theory, vol. 152, pp. 38-54, 2015, doi: 10.1016/j.jnt.2014.12.004.

[8] L. Comtet, "Advanced combinatorics. The art of finite and infinite expansions. Translated from the French by J. W. Nienhuys. Rev. and enlarged ed." Dordrecht, Holland - Boston, U.S.A.: D. Reidel Publishing Company. X, 343 p. Dfl. 65.00 (1974)., 1974.

[9] M.-A. Coppo and B. Candelpergher, “The Arakawa-Kaneko zeta function." Ramanujan J., vol. 22, no. 2, pp. 153-162, 2010, doi: 10.1007/s11139-009-9205-x. 
[10] M. Kaneko, "Poly-Bernoulli numbers." J. Théor. Nombres Bordx., vol. 9, no. 1, pp. 221-228, 1997, doi: 10.5802/jtnb.197.

[11] M. Katsurada, "Complete asymptotic expansions for certain multiple $q$-integrals and $q$ differentials of Thomae-Jackson type." Acta Arith., vol. 152, no. 2, pp. 109-136, 2012, doi: 10.4064/aa152-2-1.

[12] T. Komatsu, " $q$-poly-Bernoulli numbers and $q$-poly-Cauchy numbers with a parameter by Jackson's integrals.” Indag. Math., New Ser., vol. 27, no. 1, pp. 100-111, 2016, doi: 10.1016/j.indag.2015.08.004.

[13] M. Mohammad-Noori, "Some remarks about the derivation operator and generalized Stirling numbers.” Ars Comb., vol. 100, pp. 177-192, 2011.

[14] M. Rahmani, “Generalized Stirling transform.” Miskolc Math. Notes, vol. 15, no. 2, pp. 677-690, 2014.

[15] M. Rahmani, "On p-Bernoulli numbers and polynomials.” J. Number Theory, vol. 157, pp. 350366, 2015, doi: 10.1016/j.jnt.2015.05.019.

[16] H. M. Srivastava and J. Choi, Zeta and q-zeta functions and associated series and integrals. Amsterdam: Elsevier, 2012.

Authors' addresses

M. Mechacha

USTHB, Faculty of Mathematics, BP 32, El Alia, 16111 Bab Ezzouar, Algiers, Algeria

E-mail address: mmechacha@usthb.dz

M. A. Boutiche

USTHB, Faculty of Mathematics, BP 32, El Alia, 16111 Bab Ezzouar, Algiers, Algeria

E-mail address: mboutiche@usthb.dz

M. Rahmani

USTHB, Faculty of Mathematics, BP 32, El Alia, 16111 Bab Ezzouar, Algiers, Algeria

E-mail address: mrahmani@usthb.dz

\section{Behloul}

USTHB, Department of Computer Science, BP 32, El Alia, 16111 Bab Ezzouar, Algiers, Algeria

E-mail address: behloul.djilali@gmail.com 